\title{
Eagle's Syndrome: Results of Surgical Treatment
}

\author{
Weinheimer WJ and Miller FR* \\ Department of Otolaryngology Head Neck Surgery, \\ University of Texas Health Science Center at San Antonio, \\ USA \\ *Correspondling author: Frank R Miller, Department \\ of Otolaryngology Head Neck Surgery, University of \\ Texas Health Science Center at San Antonio, San Antonio, \\ USA
}

Received: February 09, 2017; Accepted: March 03, 2017; Published: March 10, 2017

\section{Abstract}

Purpose: To evaluate the success of surgical removal of an elongated styloid process in patients diagnosed with Eagle's syndrome.

Methods: A case series of 8 patients treated surgical for Eagle's syndrome was reviewed with comparison of pre-operative and post-operative pain. A selected case is presented with preoperative CT scans and intraoperative images to show the extent of enlargement of the styloid that can occur.

Main Findings: In our series, $88 \%$ (7 out of 8 ) patients had a significant decrease in pain after surgical removal of the styloid process with no postoperative complications.

Principal Conclusions: Eagles syndrome is a rare cause of orofacial pain but our case series shows that in appropriately diagnosed patients surgical removal of the elongated styloid process can provide significant relief.

Keywords: Eagle's syndrome; Tonsillectomy; Styloid Process

\section{Introduction}

Eagle's syndrome is a rare disorder that results from a large, elongated styloid process causing pharyngeal pain, cervico-facial pain, odynophagia, and dysphagia. The earliest description of an ossified styloid ligament was in 1652 by anatomist Pietro Marchetti in Italy. Weinlecher is credited with first reported surgical treatment of symptoms related to an elongated styloid process in 1872 [1]. Several sporadic case reports followed; however, Dr. Watt Eagle was first to provide a comprehensive description of symptoms associated with elongation of styloid process and/or calcification of styloid ligament, published in 1937 [2,3]

Presented here is a concise review of Eagle's syndrome, the results of a series of patients with the diagnosis of Eagle's syndrome that were treated surgical, as well as radiographic imaging and intraoperative photographs of a select case depicting the extent of styloid process enlargement that can be seen. We feel that surgery can be offer and can have excellent success in properly selected patients in whom an accurate diagnosis of pain associated with an elongated styloid process and medical management of pain has failed.

\section{Materials and Methods}

After IRB approval, a retrospective case series of 8 patients (treated over a 7 year period from 2006-2013) with signs, symptoms, and imaging consistent with Eagle's Syndrome who were treated surgically was reviewed. Surgical approach, age, sex, and pre- and post- operative pain scores were recorded. The patients' pain was assessed on a scale from 1 to 10 , with a score of 1 being no pain and a score of 10 being unbearable pain. The patients' self pain assessment score was documented. A cure for the purposes of the study was defined as post-operative pain score of 3 out of 10 or less.

Patients were selected as surgical candidates by the senior author after a thorough history, complete head and neck exam, reviewing CT imaging, and failed medical management with NSAIDS.
The transcervical surgical approach was utilized in 7 of the 8 patitents. With patient's neck slightly extended, a transverse incision was mad approximately $2 \mathrm{~cm}$ below the mandible to avoid the marginal mandibular branch of the facial nerve. Subplatysmal flaps were elevated and the anterior surface of the sternocleidomastoid muscle was released up to the mastoid tip. The posterior belly of the digastric muscle was identified. The dissection was carried down to the styloid process with care taken to avoid injury the facial and hypoglossal nerves. Muscular and ligamentous attachments to the styloid process were dissected off of the bone. The styloid was then shortened to an appropriate length and the wound closed in layer fashion [4-6].

One patient underwent an intraoral approach. With this approach, the patient is preferably nasotracheally intubated. A tonsillectomy is performed if patient has not previously been performed. The styloid process is palpated and dissection is carried through the tonsillar fossa to the styloid process.

\section{Results}

Seven of the eight patients underwent a transcervical approach. Six of the eight patients were female. The average age at presentation was 47 years. Pre-operated pain scores ranged from 7 to 10 with a

Table 1: Results of pre-operative and post-operative pain.

\begin{tabular}{|c|c|c|c|c|}
\hline Age & Sex & Pre-op pain & Post-op pain & Approach \\
\hline 42 & F & 9 & 0 & Transcervical \\
\hline 46 & M & 9 & 7 & Transcervical \\
\hline 39 & M & 10 & 2 & Transcervical \\
\hline 37 & F & 7 & 1 & Transcervical \\
\hline 41 & F & 8 & 3 & Intraoral \\
\hline 56 & F & 8 & 2 & Transcervical \\
\hline 51 & F & 7 & 1 & Transcervical \\
\hline 67 & F & 8 & 3 & Transcervical \\
\hline
\end{tabular}




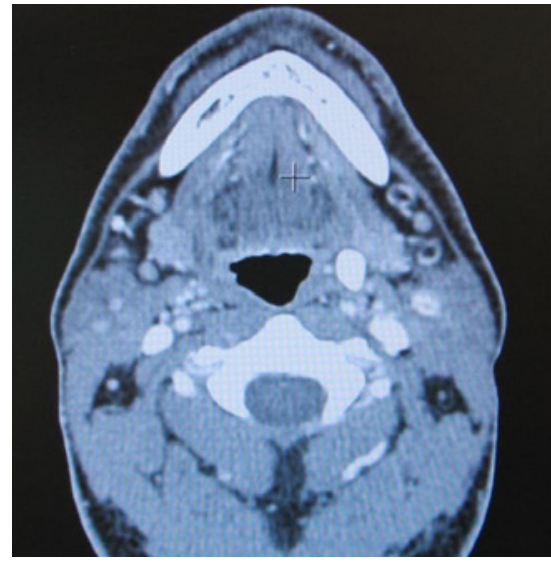

Figure 1: An axial CT scan showing a wide and long styloid is approaching the left lateral pharyngeal wall.

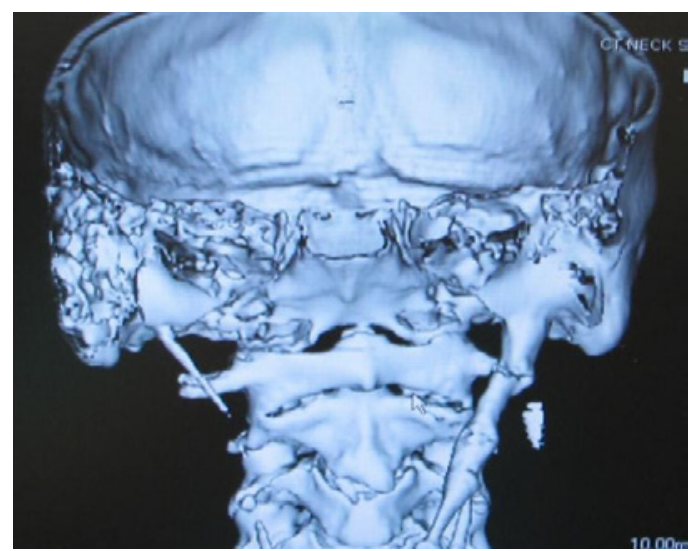

Figure 2: $\mathrm{A} 3 \mathrm{D}$ reconstruction of the same patient

median average of 8.25 .

A cure rate of $88 \%$ (7 out of 8 ) was noted. Post-operative pain scores ranged from 0 to 7 with a median of 2.25 . The patient with a post-operative pain score of 7 was a male that underwent a transcervical approach. No post-operative complications were noted (Table 1).

Imaging and intra-operative photographs are shown in Figures 1-4 of a patient who presented with significant symptoms related to a very large, elongated styloid process. The patient underwent transcervical removal and was satisfied with results, having a significant decrease in pain.

The preoperative CT scan and the $3 \mathrm{D}$ reconstruction show a very elongated, widened, finger-like projection of the left styloid process. Figures 3,4 show intraoperative photographs of the large bony projection prior to and after removal.

\section{Discussion}

The styloid process is a slender bony projection from the lower surface of the petrous portion of the temporal bone that process projects inferiorly and anteriorly into parapharyngeal space, putting in close proximity to the internal jugular vein, internal carotid artery, cranial nerves IX-XII, and the sympathetic chain [1].

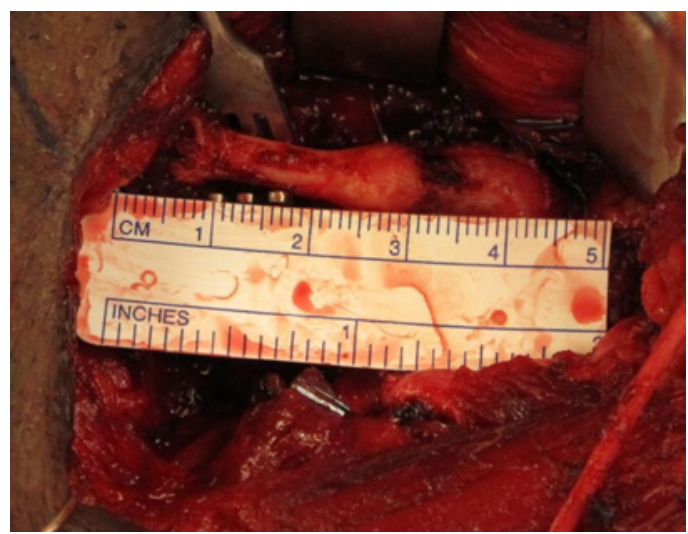

Figure 3: An intra-op photograph of the transcervical approach to the styloid process just prior to removal.

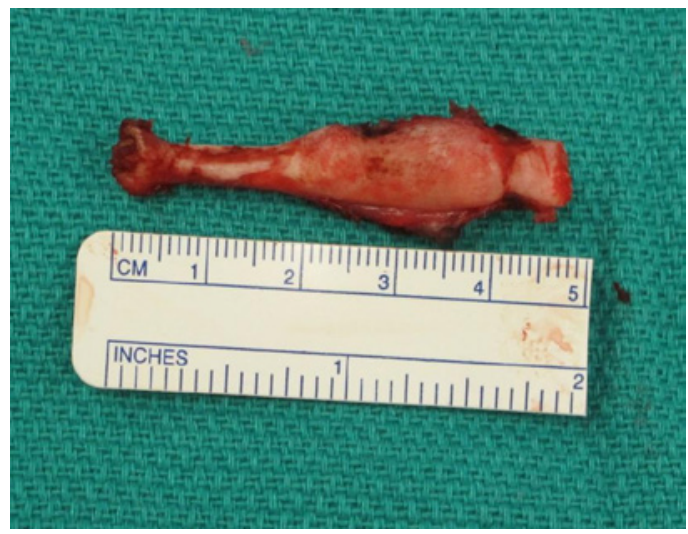

Figure 4: The excised styloid process that measures approximated $5 \mathrm{~cm}$ in length.

Most of the literature reports that greater than 2.5 to $3.0 \mathrm{~cm}$ is abnormally long $[1,5]$. The incidence varies by report. Review of literature reveals wide range of reported incidence styloid elongation from $3 \%$ to as high as $28 \%$ with most of these people being asymptomatic [4]. Incidental findings have increased with increasing radiograph use. Frequently bilateral elongation is seen, but symptoms, if present, are usually unilateral. It is important to emphasize that the mere presence of an elongated styloid process does not necessitate intervention. It is the correlation of the symptoms in conjunction with the anatomically prominent styloid process that will lead to consideration of surgical intervention.

The etiology is unclear. It is possibly an anatomic variant $v s$. post traumatic calcification. Some have reported association with posttonsillectomy patients; it is suggested to be related to granulation tissue and scar formation around the bone trapping nearby nerves [4]. Symptoms depend not only on length, but also width and projection of the styloid $[4,5]$.

Differential diagnosis for orofacial pain is broad and includes trigeminal, glossopharyngeal, occipital, and sphenopalatine neuralgia, temporomandibular joint disorders, oral, tonsil, and otologic infection, and migraine [5]. Suspicion of Eagle's Syndrome should be raised if pain is aggravated with neck flexion, extension, 
and/or rotation. An elongated styloid may be palpable through the tonsillar fossa. A CT scan of the neck is used to evaluate the size and path of the styloid and aides in making the diagnosis.

Prior to considering surgery, medical therapy is the recommended first line treatment for Eagle's Syndrome. Non-steroidal antiinflammatory drugs were attempted to all of the patients in our case series. In addition to non-steroidal anti-inflammatory drugs, other oral drugs have been used for the neurogenic pain including gabapentin, amitriptyline, valproate, and carbamazepime. Transpharyngeal injection of steroids and/or an analgesic have been used as well but only give temporary relief [8].

For those who have failed medical treatment, surgery is a viable treatment option. Two surgical approaches have been widely accepted, the transcervical (extra oral) approach and a transpharyngeal (intraoral) approach. Most literature reports that $80-90 \%$ of surgical patients will have decreased symptoms and complication rates are low [4-6].

The transcervical approach is more frequently used by the senior author. The advantages of this approach are that it allows better visualization of the nearby cranial nerves as well as the carotid artery and internal jugular vein. As opposed to the intraoral approach, the transcervical approach can be done in sterile fashion leading to a lower potential risk of infection. The disadvantages include an external scar and risk of damage to marginal mandibular nerve $[5,6]$.

The intraoral approach is also an option. The advantages of this approach include avoidance of a scar and it may be less time consuming [5]. The disadvantages of the intraoral approach are the possible higher risk of infection, decreased ability to fully resect the styloid process back to the skull base and decreased visualization of critical adjacent structures (particularly the carotid artery and cranial nerves) [5-7].

Overall the patients in our study had good results regardless of the technique used. The one patient who failed to improve with surgery was subsequently referred to neurosurgery and underwent radiofrequency ablation of the glossopharyngeal nerve with complete resolution of pain. Radiofrequency ablation as well as microvascular decompression techniques are occasionally used to treat neuropathic pain related to the trigeminal or glossopharyngeal nerve [9].

\section{Conclusion}

Eagle's syndrome is a rare condition characterized by pharyngeal pain, odynophagia, dysphagia, and occasional otalgia resulting from an elongated styloid process. As seen by the images provided, the styloid process can become impressive enlarged.

Surgical reduction in the styloid process is the usual treatment for notably symptomatic patients and can be carried out via a transcervical or an intraoral approach. Our case series of 8 patients demonstrated an $88 \%$ cure rate with a cure rate defined as a postoperative pain score of 3 out of 10 or less. No complications were noted. Surgery offers substantial improvement in pain for properly screened and selected patients.

\section{References}

1. Rechtweg J, Wax M. Eagle's Syndrome: A review. Am J Otolaryngo. 1998; 19: 316-321.

2. Eagle W. Elongated styloid process: Report of Two Cases. Arch Otolaryngol. 1937; 25: 584

3. Buono U, Mangone G, Michelotti A, Longo F, Califano L. Surgical approach to the stylohyoid process in Eagle's Syndrome. J Oral Maxillofac Surg. 2005; 63: 714-716

4. Piagkou M, Anagnostopoulou S, Koulagkouros K, Piagkos G. Eagle's Syndrome: A Review of the Literature. Clinical Anatomy. 2009; 22: 545-558.

5. Strauss M, Zohar Y. Elongated styloid process syndrome: Intraoral versus external approach for styloid surgery. Laryngoscope. 1985; 95: 976-979.

6. Chase D, Zarmen A, Bigelow W, McCoy J. Eagle's syndrome: A comparison of intraoral versus extraoral surgical approaches. Oral Surg. 1986; 62: 625629

7. Chrcanovic B, Custódio A, Felix de Oliveira D. An intraoral surgical approach to the styloid process in Eagle's syndrome. Oral Maxillofac Surg. 2009; 13 : 145-151.

8. Fusco DJ, Asteraki S, Spetzler RF. Eagle's syndrome: embryology, anatomy, and clinical management. Acta Neurochir. 2012; 154: 1119-1126.

9. Emril DR, Ho KY. Treatment of trigeminal neuralgia: role of radiofrequency ablation. J Pain Res. 2010; 3: 249-254.
Austin J Otolaryngol - Volume 4 Issue 1 - 2017

ISSN : 2473-0645 | www.austinpublishing group.com

Miller et al. (C) All rights are reserved
Citation: Weinheimer WJ and Miller FR. Eagle's Syndrome: Results of Surgical Treatment. Austin J Otolaryngol. 2017; 4(1): 1087. 